\title{
INTERVENCIÓN PSICOSOCIAL EN EMERGENCIAS Y DESASTRES DESDE LA CAJA COSTARRICENSE DEL SEGURO SOCIAL: LA EXPERIENCIA EN OSA POR EL IMPACTO DE LA TORMENTA NATE, OCTUBRE 2017
}

\section{PSYCHOSOCIAL INTERVENTION IN EMERGENCIES AND DISASTERS FROM THE COSTA RICAN SOCIAL SECURITY INSTITUTE: THE EXPERIENCE IN OSA DUE TO THE IMPACT OF THE NATE STORM, OCTOBER 2017}

\section{Nancy Navarro Bartels *}

\section{RESUMEN}

El presente artículo recupera los principales resultados de una intervención psicosocial desde la Caja Costarricense del Seguro Social, realizada en la atención de la emergencia por la inundación generada en la Tormenta Nate, en el cantón de Osa, en las fechas del 5 y 6 de octubre de 2017. La intervención se llevó a cabo en el Hospital Tomás Casas Casajús y en el Área de Salud Osa. En el texto se rescatan los elementos técnicos y operativos de la atención psicosocial de la emergencia.

PALABRAS CLAVE: INTERVENCIÓN PSICOSOCIAL * EMERGENCIA * TRABAJO SOCIAL * SALUD MENTAL

\section{ABSTRACT}

This report recovers the main results of a psychosocial intervention from the Costa Rican Social Security Institute; carried out in the attention of the emergency due to the flood generated in the Nate Storm on October 5-6, 2017, in the canton of Osa.The intervention was carried out in the Tomás Casas Casajús Hospital and in the Osa Health Area. The report recovers the technical and operative elements of the psychosocial care of the emergency.

KEYWORDS: PSYCHOSOCIAL INTERVENTION $*$ EMERGENCY $*$ SOCIAL WORK * MENTAL HEALTH 


\section{INTRODUCCIÓN}

La tormenta Nate que afectó al cantón de Osa en las fechas del 5 y 6 de octubre de 2017, en términos generales, dejo secuelas y daños a familiares y funcionarios de la Caja Costarricense del Seguro Social. En este contexto, las autoridades locales solicitaron apoyo desde la Dirección Regional de Servicios de Salud Brunca a Oficinas Centrales de la Caja Costarricense del Seguro Social (ccss), las acciones se articularon con el Centro de Atención de Emergencias y Desastres (CAED), desde donde se desplegó la movilización de un equipo interdisciplinario psicosocial a la zona, con el objetivo de realizar una valoración y clasificación de los casos prioritarios para la atención psicosocial desde la salud de las personas funcionarias institucionales.

Desde lo operativo, se desarrollaron las acciones según el protocolo institucional "Lineamiento para la atención de emergencias y desastres desde Trabajo Social del Seguro de Salud de la CCSS", de noviembre de 2016 (código LGM.DDSS-ARSDT-CNTS.071116). En específico, el protocolo incluye las determinaciones técnicas para profesionales en Trabajo Social de la institución en la atención de los desastres y emergencias en las diferentes etapas (desde las primeras 72 horas, de las 72 horas a los 3 meses $y$ posterior a los 3 meses).

La intervención psicosocial implicó en términos generales un trabajo en equipo interdisciplinario y se llevó a cabo del 24 al 27 de octubre de 2017, en el Hospital Tomás Casas Casajús y en el Área de Salud Osa, momento en el cual la zona Sur se mantenía aún en alerta amarilla por las condiciones climáticas.

El presente texto tiene como fin último ser un insumo $y$ aporte al gremio profesional en la atención de emergencias y desastres con implicaciones psicosociales, desde los elementos técnicos, operativos y de logística.

\section{LA EMERGENCIA: AFECTACIÓN DE LA TORMENTA NATE}

Según el periódico de circulación nacional La Nación, reporta en los sucesos del 5 de octubre de 2017, "Inundaciones, derrumbes, comunidades aisladas, afectados en albergues son las consecuencias visibles, por el momento, de los efectos indirectos de la tormenta tropical Nate en Costa Rica" (p.1).

Según datos de la Universidad de Costa Rica (2017) por la emergencia se habilitaron 178 albergues, para atender a 11361 personas en el momento más crítico del evento en el país. En cuanto a las personas rescatadas durante los días intensos de lluvias se reporta:

$\diamond \quad 1500$ por el Cuerpo de Bomberos.

$\diamond \quad 1021$ por la Cruz Roja Costarricense.

$\diamond \quad 20$ vuelos ambulancias con 89 personas.

Se realizó un gasto de $\$ 2350$ millones en inversión con recursos de la Comisión Nacional de Emergencias (CNE) solo en los primeros días.

$\diamond \quad$ Asistencia humanitaria.

$\diamond \quad$ Materiales y suministros.

$\diamond \quad$ Primeros impactos en comunidades afectadas.

Hasta el 12 de octubre se había entregado 16144 diarios por la Fuerza Pública en 79 comunidades. A nivel nacional fue catalogado como uno de los desastres más graves en los últimos tiempos, según el Periódico El País (2017):

El Gobierno de Costa Rica calificó este lunes la tormenta tropical Nate como el peor desastre natural que ha impactado al país en décadas debido a los severos daños que causó en agricultura, infraestructura $y$ viviendas en tres cuartas partes del país.

"Nate es de los desastres naturales más grandes que ha vivido el país en las últimas décadas, sus efectos son más grandes que los del huracán Otto", que impactó directamente a Costa Rica en noviembre de 2016, dijo en una conferencia de prensa la vicepresidenta Ana Helena Chacón, encargada de la coordinación política de la emergencia (p.1). 
Los daños de la emergencia a la zona Sur $y$ otras comunidades fueron de impacto nacional, durante el evento, como en los días y semanas posteriores, lo que corresponde a la etapa inicial e intermedia. Según la Organización Panamericana de la Salud (oPs, 2009) en emergencias se definen tres etapas del proceso desde el evento hasta los días o semanas posteriores:

$\diamond \quad$ Etapa inicial: se efectúa en las primeras 72 horas posteriores al evento.

$\diamond \quad$ Etapa intermedia: posterior al evento después de las primeras 72 horas hasta los 30 días; registra la evolución de la situación de salud mental en forma continua durante las primeras cuatro semanas.

$\diamond \quad$ Etapa final: se lleva a cabo al concluir la etapa intermedia.

Específicamente, en el caso de la atención de la emergencia de Osa se realizó en la etapa intermedia, 19 días post-evento, la cual se describe como:

... un proceso sistemático y más detallado de recolección de información, aproximadamente durante el primer mes, lo que contribuye a definir el plan de intervenciones en ese periodo. Hace un análisis de los daños a la salud mental, las necesidades psicosociales y los factores de riesgo, y las acciones que se están realizando (oPs, 2009, p. 15).

En esta etapa intermedia, desde el trabajo de campo comunitario (martes 24 de octubre de 2017 en horas de la tarde), por medio de la técnica de observación, se evidencian los daños de la emergencia por la inundación en la zona asociada al desbordamiento del Río Térraba.
FOTOGRAFÍA 1

VIVIENDAS EN CIUDAD CORTÉS CON AFECTACIÓN TORMENTA NATE, OCTUBRE 2017

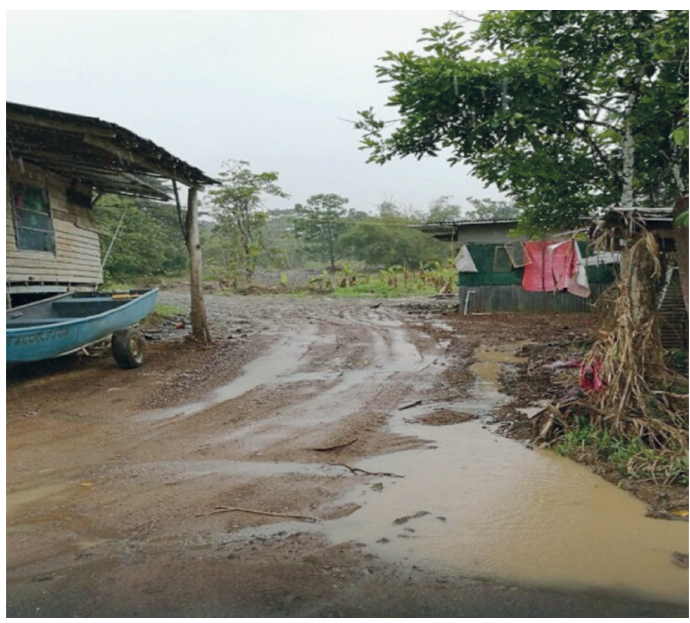

Fuente: Tomada por la autora, octubre 2017.

FOTOGRAFÍA 2

DAÑOS EN CAMINOS Y EN CULTIVOS DE LA ZONA DE CIUDAD CORTÉS

TORMENTA NATE, OCTUBRE 2017

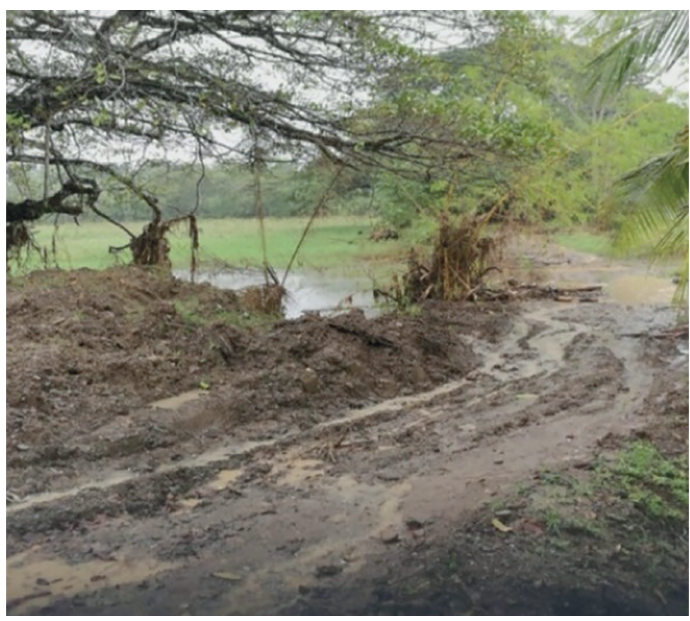

Fuente: Tomada por la autora, octubre 2017. 
FOTOGRAFÍA 3

PUENTE SOBRE EL RÍO TÉRRABA, UBICADO

ENTRE LA ZONA DE PALMAR NORTE

Y PALMAR SUR

TORMENTA NATE, OCTUBRE 2017

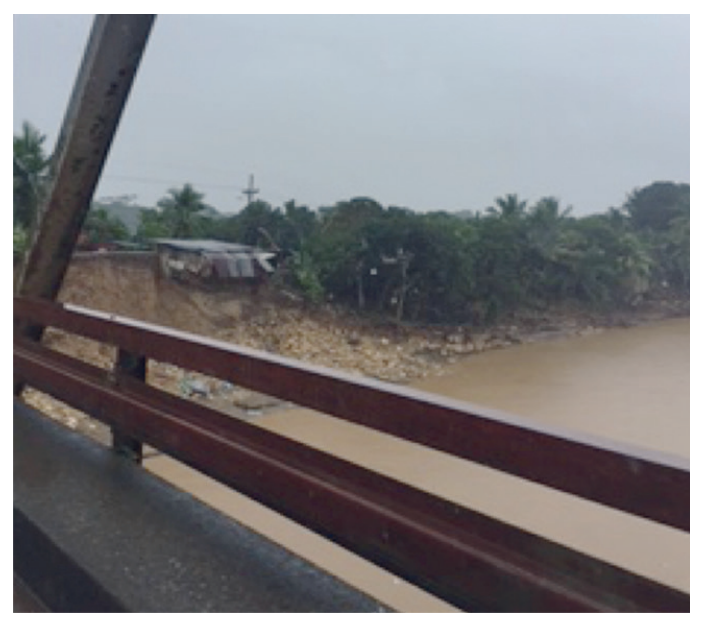

Fuente: Tomada por la autora, octubre 2017.

En términos generales, en la comunidad de Osa se evidencia el impacto de la inundación en la zona con afectaciones, principalmente en: viviendas, comercios, cultivos, carreteras, infraestructura institucional, medios de transporte, entre otros.

Por lo general, los desastres tienen consecuencias económicas, producen devastación, empobrecimiento, destrucción ambiental y de la infraestructura, $y$ carencia de servicios básicos como agua potable y alimentos; pero, también, producen un deterioro en la vida de las personas y una desintegración de las familias y de la comunidad (oPs, 2009, p.1).

En este sentido, la afectación en la zona trasciende lo material e incluye aspectos psicosociales $y$ de la salud mental de las personas $y$ familias que residen en la comunidad.

Los efectos de los desastres sobre la salud física suelen ser bastante conocidos, con secuelas a corto, mediano y largo plazo. En cambio, no siempre se han reconocido de igual manera los efectos sobre la salud mental, a pesar de haberse demostrado que, en situaciones de desastres $y$ emergencias complejas, se produce un incremento de los signos de sufrimiento psicológico, como la aflicción y el miedo; se puede aumentar en cierta medida la morbilidad psiquiátrica y otros problemas sociales. Se estima que entre una tercera parte y la mitad de la población expuesta (según la magnitud del evento $y$ otros factores) sufre alguna manifestación psicológica; aunque debe destacarse que no todas pueden calificarse como patológicas, la mayoría debe entenderse como reacciones normales ante situaciones de gran significación o impacto. También, se ha demostrado que, después de la emergencia propiamente dicha, los problemas de salud mental en los sobrevivientes requieren de atención durante un periodo prolongado, cuando tienen que enfrentar la tarea de reconstruir sus vidas (OPS, 2009, p. 5-6)

\section{UNA APROXIMACIÓN A LA ATENCIÓN PSICOSOCIAL DE LA EMERGENCIA}

Para la comprensión de la emergencia, desde la perspectiva psicosocial, se visualiza como:

... un hecho súbito, inesperado, indeseable $y$, generalmente, imprevisible que, cuando menos, interrumpe y altera la rutina cotidiana de la/s persona/s directamente afectada/s, de su entorno cercano (familia, vecinos, etc.) y/o de una colectividad (grupo, pueblo, ciudad, etc.); requiriendo su resolución (la de sus diferentes problemas), con frecuencia, actuaciones urgentes (Arricivita, 2012, p. 19).

En tanto, las emergencias y los desastres son circunstancias que generan condiciones de crisis en un espacio y tiempo determinado, con un impacto material $y$ humano, generando necesidades asistenciales $y$ de atención psicosocial por el efecto directo en la salud mental de las personas. 
Las situaciones de emergencia generan multitud de problemas y necesidades (sanitarios, de rescate, de seguridad, sociales, etc.). Desde hace unos años ha cobrado un interés significativo, sobre el resto de actuaciones, la intervención psicosocial en emergencias y catástrofes; tanto en España como en espacios internacionales (Arricivita, 2012, p.18).

Según la ops (2009), "el impacto psicosocial de cualquier evento traumático depende de factores como la naturaleza misma del evento, las características de la personalidad de las víctimas, el entorno y las circunstancias" (p.3). Estos elementos deben considerarse como interrelacionados desde la intervención.

Siguiendo al autor Arricivita (2012), "lo psicológico y lo social, de las personas afectadas por una experiencia estresante o traumática, no puede entenderse por separado, como si cada uno de ellos fuera un añadido del otro. Sólo puede entenderse como un todo: un modo de entender y de proceder" (p.20). Por lo anterior, el apoyo psicosocial como un conjunto es vital para dar respuesta a las necesidades surgidas en la emergencia, siguiendo esta perspectiva se comprende esta categoría como un:

Proceso de acompañamiento $y$ seguimiento articulado, interdisciplinario, interinstitucional, intersectorial y comunitario basado en la gestión del riesgo, orientado a restablecer la cotidianeidad de las personas, la integridad emocional y reactivar sus redes sociales, con un enfoque participativo, de derechos y de género, brindado por personal especializado y no especializado (Comité Asesor Técnico de Apoyo PsicosocialCATAPS, 2010).

Desde la experiencia nacional, según $\mathrm{Fa}$ llas y Valverde (2016) indican que:

En Costa Rica se han realizado esfuerzos aislados por incorporar la atención psicosocial en esos procesos de emergencias y desastres, que aunque han sido exitosos carecen de una estructura organizativa que integre el trabajo de diferentes disciplinas e instituciones, además de ello se centra en la respuesta, sin considerar la reducción de la vulnerabilidad, la mitigación, la preparación y la recuperación de la salud mental.

Uno de los sectores que interviene en situaciones de emergencias $y$ desastres es el sector salud, en el que se destaca la cCss, institución prestadora de servicios de salud a la población, que incluye la protección y cuidado de la salud mental, por parte de profesionales en Medicina, Enfermería, Psicología y Trabajo Social (p. 257).

Desde el Trabajo Social, la intervención psicosocial es una acción profesional sustantiva desde el sector salud, por las implicaciones que tienen en la salud integral de las personas, en especial desde la perspectiva de la salud mental.

La respuesta institucional en el campo de la salud mental es compleja, ya que no solamente se trata de atender las consecuencias emocionales directas del evento (miedo, ansiedad, tristeza, rabia, etc.), sino, también, otros efectos indirectos en la dinámica interpersonal y social, así como en el entorno de las víctimas, en la medida en que se produce un deterioro del tejido social y una desestructuración de la vida familiar (oPS, 2009, p. 6).

Según la experiencia recuperada por $\mathrm{Fa}$ llas y Valverde (2016) en la ccss, la disciplina de Trabajo Social ha estado presente en la atención psicosocial en diferentes eventos que impactan la salud de la población, entre ellas se destacan las experiencias de:

$\diamond \quad$ Incendio del Hospital Dr. Calderón Guardia, 2005.

$\diamond \quad$ Terremoto de Cinchona (Central Norte), 2009.

$\diamond \quad$ Deslizamiento en Barrio Lajas de Escazú (Valle Central), 2010.

$\diamond \quad$ Deslizamientos en Aserrí y Acosta (Valle Central), 2010. 
$\diamond \quad$ Inundaciones en Parrita (Pacífico Sur), 2008 y 2010.

$\diamond \quad$ Terremoto de Nicoya (Pacífico Norte), 2012.

$\diamond \quad$ Deslizamiento en Poasito como secuela del Terremoto de Cinchona (Central Norte), 2013.

$\diamond \quad$ Inundación en Turrialba (Central Sur), 2015.

$\diamond \quad$ Erupciones del Volcán Turrialba (Central Sur), 2010.

En la intervención se ha utilizado como base el "Lineamiento para la atención de emergencias y desastres desde Trabajo Social del Seguro de Salud de la ccss", en donde se establece que la función principal de la ccss y por ende de Trabajo Social es la atención de las personas afectadas por emergencias $y$ desastres, sus familias y otros recursos de apoyo, que acuden a los centros de salud, así como, al personal de la Institución.

En el caso específico de la emergencia de Osa, se aplicó este lineamiento y para ello, se conformó un equipo operativo con perfil psicosocial que estuvo integrado por Trabajadoras Sociales, Psicólogas y Enfermeras en Salud Mental, entre ellas:

$\diamond \quad$ MSc. Jessica Pérez Villalobos, Enfermera Salud Mental, Hospital Dr. Rafael Ángel Calderón Guardia.

$\diamond \quad$ MSc. Viviana Solórzano Arias, Psicóloga, Área de Salud de Parrita.

$\diamond \quad$ MSc. Marvel Torres Córdoba, Trabajadora Social, Jefe Hospital Ciudad Neily.

$\diamond \quad$ MSc. Damaris Chavarría Fernández, Enfermera Salud Mental, Hospital San Juan de Dios.

$\diamond \quad$ Licda. Denia Montero Porras, Psicología, Hospital de Ciudad Neily.

$\diamond \quad$ Licda. Evelyn Vega Guido, Trabajadora Social, Hospital Ciudad Neily.

$\diamond \quad$ MSc. Alejandra Salazar Jiménez, Psicóloga, Área de Salud Esparza.

$\diamond \quad$ Licda. Graciela Arguedas Monge, Trabajadora Social Supervisora Regional de Servicios de Salud Brunca.

$\diamond \quad$ MSc. Nancy Navarro Bartels, Trabajadora Social, Clínica Clorito Picado/Líder del equipo operativo.
En términos generales, la intervención psicosocial debe ser interdisciplinaria, según Arricivita (2012), la interdisciplinariedad es la línea de intervención en emergencias colectivas y en catástrofes, donde participan diversos profesionales como trabajadores sociales, psicólogos, personal sanitario, etc. De la misma manera, López (s.f) indica que "las Emergencias Sociales son un campo de intervención multidisciplinar en el que trabajadores/as sociales, psicólogos/as, educadores/as sociales - así como otros profesionales psicosociales - desarrollan su actividad profesional de forma coordinada" (p.11).

\section{ESTRATEGIA PARA LA ATENCIÓN PSICOSOCIAL DE LA EMERGENCIA}

La estrategia se centró en la valoración $y$ atención psicosocial inicial por la afectación de la emergencia, esto desde el trabajo interdisciplinario, como corresponde en una etapa intermedia. Desde la visión de Herrero (2011), se trabaja sobre la urgencia, la severidad de los riesgos inmediatos, la detección de casos que requieran de intervención específica y de la capacidad de afrontamiento.

En este sentido, desde lo técnico de la atención de la emergencia de Osa, la estrategia psicosocial se centró en:

$\diamond \quad$ Desde la valoración, identificar y clasificar los riesgos inmediatos y los recursos disponibles de afrontamiento a nivel personal, familiar, comunal e institucional.

$\diamond \quad$ Desarrollar acciones en los casos necesarios de intervención en crisis o auxilios psicológicos.

$\diamond \quad$ Brindar orientación en cuanto a las herramientas de manejo o afrontamiento con respecto a los recursos disponibles en cada particular.

$\diamond \quad$ Referir a los servicios de salud necesarios.

$\diamond \quad$ Integración de un informe psicosocial.

Es vital que posterior a la atención de equipo interdisciplinario, se gestionen para el seguimiento los planes de afrontamiento, los cuales se incluyen en las referencias a los servicios de salud según las necesidades detectadas. En el caso específico de la experiencia en Osa, 
se incluyeron los servicios de Trabajo Social, Psicología, Psiquiatría y Medicina laboral. Todos los servicios antes mencionados corresponden a los mismo ccntros de salud intervenidos, a excepción de un caso en el que se realizó una referencia al equipo EISAN de Ciudad Neily

Como parte de la sistematización de la atención de la emergencia, se realizaron dos informes psicosociales; dirigidos a la Dirección
Médica de cada centro de salud para el seguimiento, específicamente al Hospital Tomás Casas Casajús y al Área de Salud Osa. Además, fue socializado posteriormente en una reunión efectuada el 16 de noviembre de 2017 con los contactos del Centro de Atención de Emergencias y Desastres (CAED). La estrategia antes descrita se sintetiza en el flujograma de la figura 4:

FIGURA 1

ESTRATEGIA PSICOSOCIAL EN CASO DE EMERGENCIA Y DESASTRES

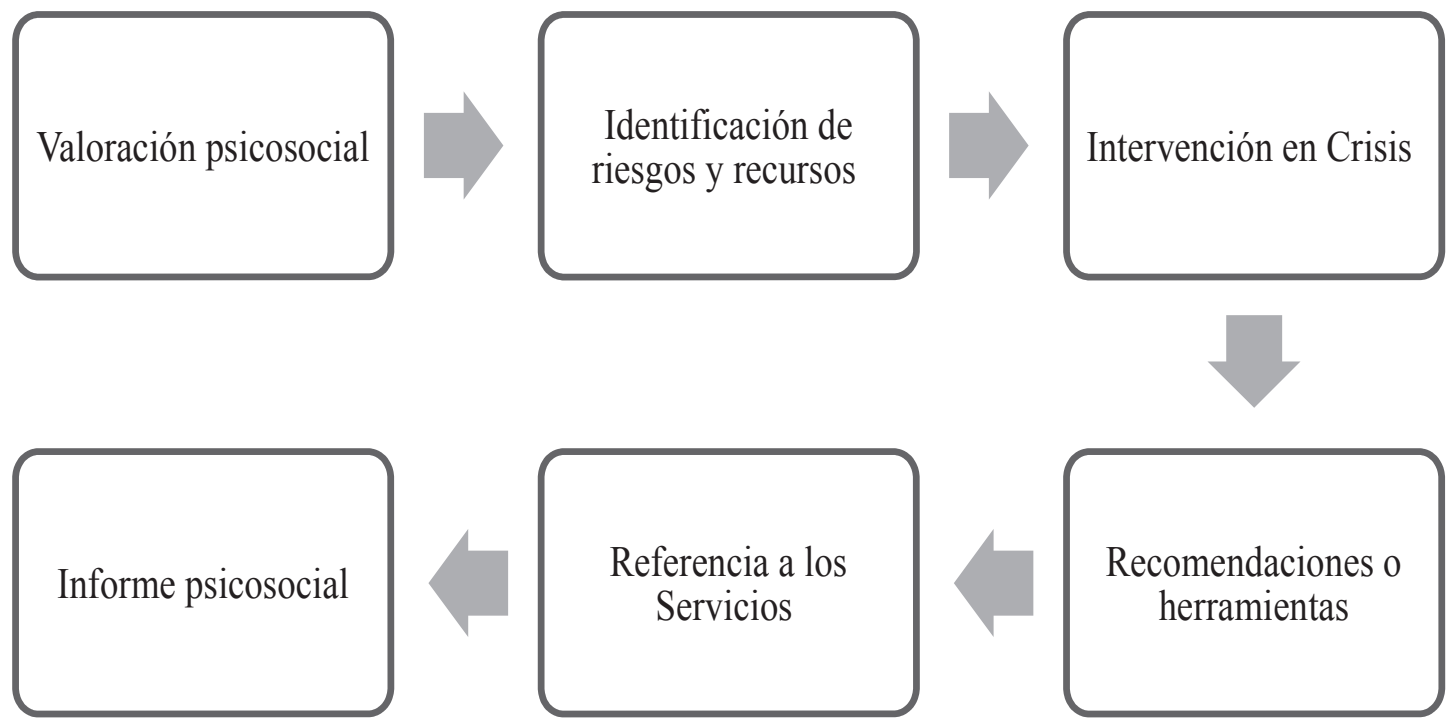

Fuente: Elaboración propia.

Para el flujo exitoso de este proceso que permita el cumplimiento de la valoración psicosocial y atención inicial de la emergencia, se requiere de acciones profesionales coordinadas, criterios interdisciplinarios comunes en el equipo de trabajo operativo y de un liderazgo con habilidades en:

$\diamond \quad$ Estilo de liderazgo positivo.

$\diamond \quad$ Capacidad técnica en el manejo de emergencias y desastres.

$\diamond \quad$ Capacidad en la toma de decisiones bajo situaciones de emergencia. $\diamond \quad$ Conocimiento de los instrumentos de valoración psicosocial y familiar.

$\diamond \quad$ Capacidad para la articulación logística de la emergencia desde el centro de salud o la comunidad específica.

$\diamond \quad$ Articulación de criterios técnicos y dirección de sesiones de equipo para la discusión de casos.

$\diamond \quad$ Conocimiento de la clasificación TRIAGE psicosocial.

$\diamond \quad$ Administración del tiempo y los recursos del equipo operativo. 
Históricamente, el gremio profesional de las trabajadoras sociales ha desarrollado habilidades para liderar procesos logísticos y equipos interdisciplinarios, con un alto compromiso $y$ calidad en la compresión de los elementos técnicos que conlleva el manejo de una emergencia con implicaciones en el área psicosocial.

Según el "Lineamiento de atención de emergencias $y$ desastres desde Trabajo Social del Seguro de Salud de la ccss", la disciplina es pionera en la atención psicosocial a personas usuarias y funcionarias; así como, sus familias, en la aplicación de instrumentos y en la sistematización de las experiencias.

Asimismo, profesionales en Psicología o en especialidades de la salud mental desempeñan estas labores psicosociales para la atención de emergencias, debido a sus habilidades profesionales en esta área. En común es relevante que el líder y el equipo operativo tengan habilidades y conocimientos técnicos para la aplicación de los instrumentos disponibles en la atención del evento, en especial los que se incluyen como parte del protocolo institucional para el manejo de la atención de emergencias y desastres.

\section{INSTRUMENTOS PARA LA ATENCIÓN DE EMERGENCIAS PSICOSOCIALES Y ELEMENTOS DEL TRIAGE PSICOSOCIAL}

El principal objetivo de los instrumentos es evaluar de manera objetiva el impacto psicosocial de la emergencia.

Cuando hablamos de impacto psicosocial, nos referimos a los efectos que generan los desastres en el ámbito psicológico individual, familiar y social de las víctimas. Estos efectos se relacionan con muchas variables, entre las que se encuentran las condiciones de vida de la persona y el grado de deterioro de su ambiente físico y social (oPs, 2009, p.1).

Desde los lineamientos institucionales de la ccss se establecen los instrumentos a utilizar para la valoración psicosocial en caso de emergencias, entre ellos: 1) Instrumento Evaluativo CASIC (CASICFLE) y 2) Instrumento de Valoración Familiar.

En la aplicación de los instrumentos, se identifican las principales áreas afectadas en la persona y grupo familiar, así como, se rescata desde el criterio profesional psicosocial, la clasificación para priorizar la atención, esto como una estrategia para determinar las recomendaciones específicas para el seguimiento.

Estos instrumentos evalúan la afectación en las personas por la emergencia desde diversas áreas internas y externas, a nivel conductual, afectiva, interpersonal, somática, entorno, cognoscitiva, familiares y laboral.

En la aplicación de la Valoración Familiar desde la clasificación del TRIAGE psicosocial (incluido en el anexo 3 del "Lineamiento para atención de las emergencias y desastres desde Trabajo Social"); el instrumento permite determinar y priorizar las valoraciones, con una identificación de rojos (alto riesgo), amarillos (riesgo moderado o con recursos de apoyo) y verdes (bajo o ningún riesgo).

Desde la estrategia de intervención en la emergencia de Osa, el equipo operativo psicosocial determinó las siguientes categorías en el TRIAGE (Tabla 1). 


\section{CUADRO 1}

TRIAGE PSICOSOCIAL EN LA EXPERIENCIA DE OSA

\begin{tabular}{|c|c|c|}
\hline CATEGORÍA & INDICADORES & PLAN DE TRATAMIENTO PSICOSOCIAL \\
\hline ROJOS & $\begin{array}{l}\text { Agudización de dinámicas de violencia } \\
\text { intrafamiliar y/o problemas relacionales. Sin } \\
\text { redes de apoyo efectivas ante la emergencia. } \\
\text { Grupo familiar con adaptaciones posterior a } \\
\text { estancia en albergue. } \\
\text { Síntomas depresivos que pueden generar } \\
\text { crisis o riesgos para la vida. } \\
\text { Integración de miembros familiares con } \\
\text { necesidades especiales por dependencia } \\
\text { funcional. }\end{array}$ & $\begin{array}{l}\text { Estos casos en la emergencia atendida se } \\
\text { sugieren que deben ser atendidos con un } \\
\text { criterio de prioridad, en los Servicios de } \\
\text { Psiquiatría, Psicología y Trabajo Social, de } \\
\text { acuerdo a la necesidad de cada caso. }\end{array}$ \\
\hline $\begin{array}{l}\text { AMARILLO/ } \\
\text { SEGUIMIENTO }\end{array}$ & $\begin{array}{l}\text { Identificación de sentimientos de intran- } \\
\text { quilidad, ansiedad, labilidad emocional, etc. } \\
\text { Sintomatología física somática. } \\
\text { Dinámica familiar con afectación. }\end{array}$ & $\begin{array}{l}\text { Afectación material y psicosocial que } \\
\text { requieren de seguimiento. } \\
\text { Referencia a los servicios de Psiquiatría, } \\
\text { Psicología y Trabajo Social, de acuerdo a la } \\
\text { necesidad de cada caso. }\end{array}$ \\
\hline $\begin{array}{l}\text { AMARILLOS/ } \\
\text { ALTA }\end{array}$ & $\begin{array}{l}\text { Afectación material y psicosocial pero con } \\
\text { recursos de afrontamiento (personales, } \\
\text { familiares, comunales, institucionales, etc). }\end{array}$ & $\begin{array}{l}\text { Sin referencias a servicios. Se les brindó } \\
\text { recomendaciones y herramientas psicoso- } \\
\text { ciales en la atención de valoración para el } \\
\text { afrontamiento. }\end{array}$ \\
\hline
\end{tabular}

Fuente: $\quad$ Elaboración propia, 2017.

Cabe destacar, no se utilizó la categoría verde ya que a lo interno del centro de salud en Osa, se había realizado un filtro inicial, con la construcción de un listado de las personas que habían manifestado a la jefatura directa de cada servicio, estar afectadas a nivel psicosocial por la emergencia de la Tormenta Nate, lo que se visualiza como un primer esfuerzo de categorización.
SÍNTESIS DE INTERPRETACIÓN DE LOS PRINCIPALES HALLAZGOS Y RESULTADOS DE LA INTERVENCIÓN PSICOSOCIAL EN LA EMERGENCIA DE OSA

1) El proceso de atención psicosocial se desarrolló con un total de 28 funcionarios del Hospital Tomás Casas Casajus. Los funcionarios se desempeñan a nivel laboral en: Servicios Generales, Enfermería, Nutrición, Registros Médicos y en el Área de Gestión Bienes y Servicios. Se clasificaron según el triage psicosocial de la siguiente manera:

$\begin{array}{ll}\diamond & 6 \text { Rojos } \\ \diamond & 7 \text { Amarillos/seguimiento } \\ \diamond & 15 \text { Amarillos/alta }\end{array}$




\title{
GRÁFICO 1 \\ CLASIFICACIÓN TRIAGE \\ HOSPITAL TOMAS CASAS \\ 2017
}

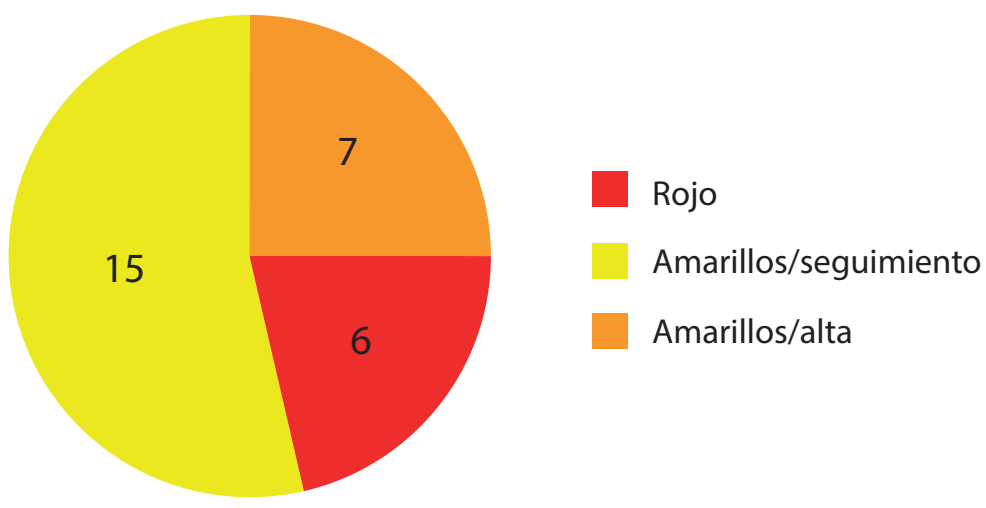

Fuente: $\quad$ Elaboración propia, 2017.

2) En el caso del Área de Salud de Osa se atendieron 8 funcionarios, que se desempeñan en: Servicios Generales, Enfermería, Registros Médicos. Se clasificaron según el TRIAGE psicosocial de la siguiente manera:

$\diamond \quad 2$ Rojos

$\diamond \quad 4$ Amarillos/seguimiento

$\diamond \quad 2$ Amarillos/Alta

\author{
GRÁFICO 2 \\ CLASIFICACIÓN TRIAGE \\ ÁREA DE SALUD OSA \\ 2017
}

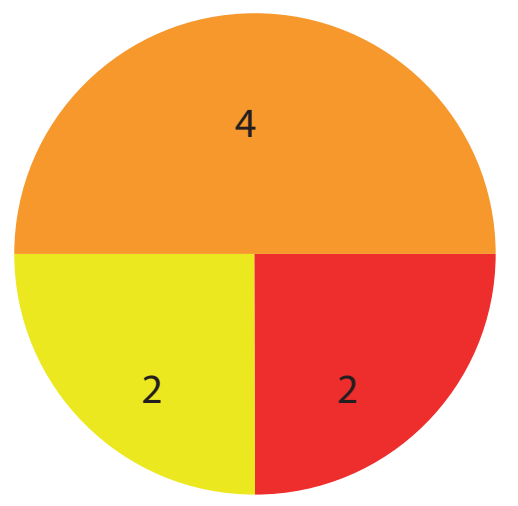

Rojo

Amarillos/seguimiento

Amarillos/alta

Fuente: $\quad$ Elaboración propia, 2017. 
3) En la totalidad de las valoraciones efectuadas, las personas reportan afectaciones patrimoniales, asociadas a daños en las viviendas por inundación de la zona, así como, afectación en los enseres (electrodomésticos, muebles u otras pertenencias).

4) Se identifican a nivel psicosocial manifestaciones en las áreas: conductual, afectiva, somática, cognitiva, familiar y laborales. Con las particularidades de cada persona según sus recursos personales, familiares, comunales, institucionales $y$ otros. En términos generales, posterior a un análisis de los hallazgos se refieren a:

$\diamond \quad$ Labilidad emocional asociada a crisis por evento.

$\diamond \quad$ Crisis de Ansiedad.

$\diamond \quad$ Duelo por pérdidas materiales o mascotas.

$\diamond \quad$ Agudización de situaciones de cuido de familiares por la crisis de emergencia (personas adultas mayores o niños).

$\diamond \quad$ Problemas en la dinámica familiar agudizados por la situación de crisis (separación u ruptura, violencia intrafamiliar, conflictivas relacionales).

$\diamond \quad$ Afectación conductual $y$ afectiva (cambios de humor, sentimientos de miedo o tristeza).

$\diamond \quad$ Síntomas somáticos (dolores musculares, trastornos de sueño).

5) A las personas detectadas con factores de riesgo o labilidad emocional, se les brindó la contención psicosocial, así como desde el criterio procesional se indicó el seguimiento requerido como prioritario.

6) Posterior a la emergencia, se reporta la participación institucional en la comunidad, entre ellos: IMAS, INAMU, Gobierno local, Comisión de Emergencias; sin embargo, al parecer en los subsidios económicos, los funcionarios y las funcionarias de la ccss no han tenido acceso debido a la condición de asalariados.

7) Desde la comunidad, indican que han percibido apoyo de grupos religioso de iglesias, así como, otros vecinos y asociaciones, en especial: alimentación, colchones, artículos de limpieza, ropa.

8) Se mantienen las manifestaciones de preocupación y angustia en las personas valoradas debido a que la zona continuaba en alerta amarilla, con medidas de prevención y precaución por los riesgos asociados.

9) En su mayoría refieren apoyo institucional por parte de las jefaturas, en lo referente a las medidas de prevención e información.

10) Las personas funcionarias fueron reubicadas por daños estructurales en el EBAIS de Cortes centro, al parecer en condiciones actuales de hacinamiento para desarrollar sus labores, lo que podría representar un factor de riesgo en las relaciones laborales entre los compañeros y compañeras.

11) Cada intervención psicosocial se incluyó en un cuadro síntesis en el Informe Psicosocial para el Hospital Tomás Casas Casajús y el Área de Salud Osa, con detalles como: datos personales, clasificación en el TRIAGE y plan o recomendación.

\section{TÉCNICA FODA DE LA ATENCIÓN PSICOSOCIAL DE LA EMERGENCIA DE OSA}

Posterior a la atención de la emergencia, se realizó un análisis de las fortalezas, oportunidades, debilidades y amenazas de la experiencia de intervención psicosocial, con el fin de definir planes de mejora o recomendaciones para experiencias futuras. Los principales resultados se incluyen en el siguiente cuadro: 


\section{CUADRO 2 \\ FODA DE LA ATENCIÓN PSICOSOCIAL TORMENTA NATE, EMERGENCIA DE OSA , 2017}

\begin{tabular}{|c|c|c|c|}
\hline FORTALEZAS & OPORTUNIDADES & DEBILIDADES & AMENAZA \\
\hline $\begin{array}{l}\text { Anuencia de un equipo } \\
\text { operativo con profesionales } \\
\text { de alta capacidad técnica } \\
\text { psicosocial, con habilidades } \\
\text { para el trabajo en equipo } \\
\text { interdisciplinario. }\end{array}$ & $\begin{array}{l}\text { Existencia de lineamiento } \\
\text { para la atención de } \\
\text { emergencias y desastres } \\
\text { desde Trabajo Social del } \\
\text { seguro de salud de la ccss. }\end{array}$ & 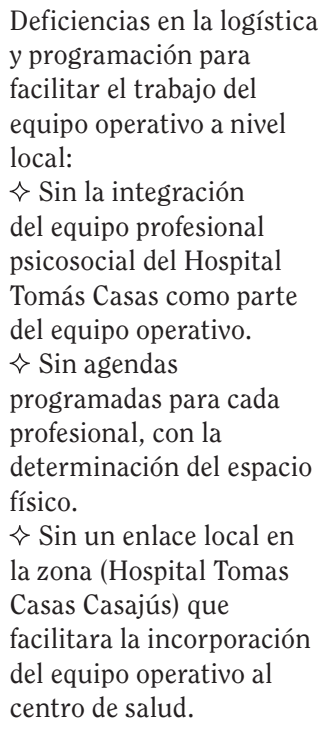 & $\begin{array}{l}\text { Persistencia al momento } \\
\text { de la atención del estado } \\
\text { de alerta amarilla por el } \\
\text { evento. }\end{array}$ \\
\hline $\begin{array}{l}\text { Cumplimiento con } \\
\text { criterios de calidad del } \\
\text { objetivo de valoración y } \\
\text { clasificación psicosocial. }\end{array}$ & $\begin{array}{l}\text { Anuencia de las regiones } \\
\text { para apoyar a nivel } \\
\text { local (Osa) en el manejo } \\
\text { de la emergencia, lo } \\
\text { que se evidencia en } \\
\text { la movilización de los } \\
\text { recursos profesionales } \\
\text { para integrar el equipo } \\
\text { operativo psicosocial. }\end{array}$ & & \\
\hline $\begin{array}{l}\text { Desarrollo de trabajo de } \\
\text { campo para ampliar el } \\
\text { criterio profesional del } \\
\text { equipo operativo. }\end{array}$ & $\begin{array}{l}\text { Apoyo del Centro de } \\
\text { Atención de Emergencias } \\
\text { y Desastres al equipo } \\
\text { operativo en la zona. }\end{array}$ & & \\
\hline $\begin{array}{l}\text { Sesiones de trabajo } \\
\text { para unificar criterios } \\
\text { profesionales posterior a } \\
\text { las valoraciones. }\end{array}$ & $\begin{array}{l}\text { Alternativas efectivas para } \\
\text { los viáticos, hospedaje y } \\
\text { transportes del equipo } \\
\text { operativo hasta la zona. }\end{array}$ & & \\
\hline \multicolumn{4}{|l|}{$\begin{array}{l}\text { Liderazgo positivo y } \\
\text { comprometido en el equipo } \\
\text { operativo, con capacidad de } \\
\text { manejo de equipo. }\end{array}$} \\
\hline $\begin{array}{l}\text { Sistematización del } \\
\text { informe psicosocial con } \\
\text { planes para el seguimiento } \\
\text { de las personas valoradas. }\end{array}$ & & & \\
\hline
\end{tabular}

Fuente: $\quad$ Elaboración propia, 2017. 


\section{CONCLUSIONES}

$\diamond \quad$ La afectación psicosocial en las personas posterior a una emergencia por desastre, tiene manifestaciones en diversas áreas desde un enfoque integral (conductual, afectiva, somática, cognitiva, familiar y laboral), sin obviar las particularidades de cada persona para su afrontamiento según sus recursos (personales, familiares, comunales, institucionales y otros).

$\diamond \quad$ La atención en salud desde la Caja Costarricense del Seguro Social contempla la intervención $y$ apoyo psicosocial a las personas afectadas por las emergencias y desastres, esto según las directrices $y$ lineamientos institucionales, con la participación de profesionales en Trabajo Social, Psicología, Salud mental, entre otros.

$\diamond \quad$ La atención de las emergencias es una intervención profesional sustantiva, que identifica la afectación psicosocial, es de índole interdisciplinaria y requiere de un líder que articule las acciones y el proceso. Según el lineamiento institucional "la intervención Trabajo Social en las emergencias $y$ desastres se caracteriza por ser parte sustantiva de la atención en salud que brinda a la población y los/as intervinientes, en las fases de preparativos y respuesta para la protección de sus derechos, su salud integral y desarrollo social" (ccss, 2016, p.14).

$\diamond \quad$ La atención psicosocial esta mediada por los elementos técnicos y logísticos, así como determinada por los lineamientos institucionales; sin embargo, no debe perder desde sus premisas que responde a las necesidades puntuales que se deriven de la emergencia en específico y de los recursos disponibles para la intervención profesional.

$\diamond \quad$ La temática de la atención psicosocial de emergencias y desastres es de relevancia para el sistema de salud costarricense y para el gremio profesional en Trabajo Social, por las secuelas que pueden significar $y$ desde una perspectiva de salud integral en las personas y sus recursos.

\section{RECOMENDACIONES}

$\diamond \quad$ La experiencia es un insumo para la atención psicosocial en emergencias $y$ desastres desde la ccss y para el gremio profesional involucrado, esta refuerza la necesidad de fortalecer las acciones de apoyo interniveles en la atención psicosocial (I, II $y$ III nivel de atención en salud), como medida ante el riesgo de otros eventos de emergencia por las condiciones de la zona.

$\diamond \quad$ En caso de necesidad de atención psicosocial por emergencia y desastre, se recomienda que los equipos de profesionales a nivel regional deben accionar los lineamientos institucionales disponibles para tales efectos. Posteriormente, según la valoración de la capacidad instalada de los servicios locales, las autoridades correspondientes pueden solicitar el apoyo a nivel de otras regiones para definir equipos interdisciplinarios de apoyo psicosocial.

$\diamond \quad$ Desde la organización jerárquica institucional en el proceso de organización y logística, se considera necesario establecer un enlace regional con el equipo operativo para favorecer la valoración psicosocial y la incorporación del equipo, que incluya dentro de sus acciones:

- Incorporación del equipo operativo en la zona afectada y en el centro de salud (es importante realizar una observación de la zona afectada para dimensionar el evento, así como incorporar al equipo operativo en el centro de salud).

- Programación de agenda de atención por profesional, según los tiempos formales estipulados para la atención psicosocial.

- Determinación de espacios físicos para la atención, con las condiciones adecuadas, accesibles, que ofrezcan privacidad.

- Disponibilidad de insumos: instrumentos de valoración, materiales de oficina, equipo de cómputo para la elaboración de informes, entre otros. 
$\diamond \quad$ En cuanto al equipo operativo es vital mantener espacios de discusión y análisis para la articulación de los criterios profesionales, previo y posteriormente a la atención, con el objetivo de favorecer en el proceso de priorización de necesidades, clasificación de TRIAGE, articulación de planes de intervención y seguimiento, elaboración de informe psicosocial, entre otros.

$\diamond \quad$ En el ámbito de recuperación, según el lineamiento, se recomienda brindar el seguimiento de las situaciones sociales de las personas afectadas orientadas a:

- Facilitar seguimiento social a las personas afectadas por la emergencia y sus familias según prioridad de atención.

- Brindar educación social para salud a las personas, familias $y$ comunidades afectadas en temas como: plan de emergencias familiar y estrategias de afrontamiento.

$\diamond \quad$ Se recomienda desde la institución, fortalecer la capacitación continua a profesionales en temas para la atención de emergencias y desastres con afectaciones psicosociales, incluir dentro de la malla curricular temas como:

- Lineamientos institucionales en el manejo de las emergencias $y$ desastres.

- Aplicación de los instrumentos de valoración psicosocial.

- Afectaciones psicosociales en las áreas: conductual, afectiva, somática, cognitiva, familiar y laborales.

- Intervención en crisis o primeros auxilios psicológicos.

Clasificación TRIAGE psicosocial.

- Trabajo en equipo bajo las circunstancias de emergencia.

- Construcción de planes familiares de emergencias.

- Liderazgo en el manejo de emergencias psicosociales.

- Elaboración de informes psicosociales de la emergencia. $\diamond \quad$ Se recomienda la sistematización de las experiencias de intervención profesional para nutrir el aporte técnico al gremio profesional, como una estrategia para enriquecer la capacidad técnica y operativa ante la atención de emergencias $y$ desastres.

\section{REFERENCIAS}

Agencia EFE (09/10/2017). Tormenta Nate es calificada por Costa Rica como el peor desastre natural en década. El País. Recuperado de http://www.elpais.com. co/mundo/tormenta-nate-es-calificadapor-costa-rica-como-el-peor-desastrenatural-en-decadas.html

Arricivita, Á. L. (2012). Intervención Psicosocial de los Trabajadores Sociales en Emergencias y catástrofes. Servicio Aragonés de Salud. Recuperado de http:// www.psicosocial-emergencias.com

Arricivita, Á. L. (2013). Atención Psicosocial en Emergencias. Red de Brigadas de Atención Psicosocial en Emergencias y Desastres. Comisión Nacional de Emergencias de Costa Rica. Recuperado de http://www.psicosocialemergencias.com

Ávalos, A. (05/10/2017). Azote de la tormenta Nate en Costa Rica. La Nación. Recuperado de https://www.nacion. com/sucesos/desastres/azote-dela-tormenta-nate-en-costa-rica/ FVOPBR6LXFFZHLIXENT3CPK53A/ story/

Caja Costarricense del Seguro Social-ccss. (2016). Lineamiento para la atención de emergencias y desastres desde Trabajo Social del Seguro de Salud de la CCSS, código LGM.DDSS-ARSDT-CNTS.071116. Área de Regulación y Sistematización de diagnóstico y tratamiento, Coordinación Nacional de Trabajo Social, Costa Rica.

Comité Asesor Técnico de Apoyo PsicosocialCATAPs. (2010). Brigada Atención Psicosocial. Apoyo psicosocial a los afectados del Huracán Otto. Escuela Psicología, Universidad de Costa Rica, Cista Rica. 
Comité Permanente entre Organismos-IAsc (2007). Guía del IASC sobre Salud Mental y Apoyo Psicosocial en Situaciones de Emergencia. Ginebra: IASC.

Corrales F., Elvia C. y Valverde N. (2016). La experiencia de la atención psicosocial en Emergencias una mirada desde la Caja Costarricense del Seguro Social. Revista El Hacer y el Pensar de la Psicología CON América Latina, II.

Herrero, I. (Diciembre 2011). Los Trabajadores Sociales en situaciones de crisis, emergencias y catástrofes. Revista Margen, (63).

López, E y Dimas, D. (s.f) La intervención del trabajadorla social en situaciones de emergencia y urgencia social. Recuperado de www.trabajosocialmalaga.org/archivos/archivos.../intervenciondelTSemergencias.pdf

Organización Panamericana de la Salud. (2009). Guía Práctica de Salud Mental en desastres. Washington, D.C.: Oficina Regional de la Organización Mundial de la Salud, Área de Preparativos para Situaciones de Emergencia y Socorro en Casos de Desastre.

Presidencia de la República de Costa Rica. (12/10/2017). Tormenta Tropical Nate entre los mayores desastres de Costa Rica. Noticias del Espacio informativo de la Presidencia de la República. Recuperado de https://www.ucr.ac.cr/ noticias/2017/10/12/tormenta-tropicalnate-entre-los-mayores-desastres-de-costa-rica.html

Universidad de Costa Rica (2017). "Tormenta Tropical Nate entre los mayores desastres de Costa Rica". Espacio informativo de la Presidencia de la República. Recuperado de https://www.ucr.ac.cr/ noticias/2017/10/12/tormenta-tropicalnate-entre-los-mayores-desastres-de-costa-rica.html

Fecha de ingreso: 03/04/2018 Fecha de aprobación: 27/09/2018 
